\title{
Diseño y proyecto como competencia transversal en la asignatura de Tecnología Hidráulica del master de Ingeniería Agronómica
}

\author{
Jaime Arviza ${ }^{a}$, Carmen Virginia Palau
}

aPDI, Dpto. Ingeniería Rural, Universitat Politècnica de València, jarviza@agf.upv.es, bPDI Dpto. Ingeniería Rural, Universitat Politècnica de València, virpaes@agf.upv.es.

\begin{abstract}
This paper presents a learning experience carried out in the subject of Hydraulics Technology in Master's Degree in Agricultural Engineering in the Universitat Politècnica de València to acquire and assess the generic competence "CT -05: Design and project" . In this sense, using a real project during the subject the students in groups of three developed in different phases the resolution of it. Teachers have tutored each of the phases until the end. The learning activity finalized by writing a final report and oral exposition of the project. The results show a good acquisition of the skill. Nevertheless, certain improvements need to be done in the teaching methodology to implement in future courses.
\end{abstract}

Keywords: generic competence, project, design, active methodologies, assessment

\begin{abstract}
Resumen
Esta comunicación presenta una experiencia docente llevada a cabo en la asignatura de Tecnología Hidráulica del Master de Ingeniería Agronómica para adquirir y evaluar la competencia transversal de "CT-05: Diseño y proyecto". Para ello, mediante un proyecto real planteado en la asignatura, los alumnos por equipos desarrollaron por fases la resolución del mismo. Cada una de las fases ha sido tutorizada por los profesores hasta finalizar con la redacción de un informe final y la defensa oral de proyecto. Los resultados muestran una buena adquisición de la competencia y se puntualizan ciertas mejoras en la metodología docente para implementar en cursos próximos.
\end{abstract}

Palabras clave: competencia transversal, proyecto, diseño, metodología activa, evaluación.

\section{Introducción}

La Universitat Politècnica de València (UPV) se ha involucrado activamente en la incorporación de ciertas competencias transversales en el currículo de sus estudiantes. De esta forma, el alumno egresado sale al mundo profesional con un valor añadido adquirido durante su periodo de formación universitaria. 
Como comenta Fernández March (2010), el concepto de competencia es víctima de su propia polisemia según los contextos y los autores. De esta forma, se define como "un saber actuar complejo que se apoya en la movilización y la combinación eficaz de una variedad de recursos internos y externos dentro de una familia de situaciones" (Tardif, 2006).

En concreto, las competencias transversales o genéricas se definen como habilidades relacionadas con el desarrollo personal, que no dependen de un ámbito temático o disciplinario especifico sino que aparecen en todos los dominios de la actuación profesional y académica (González y Wagenaar, 2003)

Para ello, la UPV ha elaborado un proyecto institucional donde se establece la estrategia de adquisición y evaluación de estas competencias (Proyecto Institucional UPV, 2015). En él, se definen 13 competencias transversales a trabajar tanto a nivel de grado como a nivel de master. Éstas deben incorporarse a la formación de los estudiantes en dos vías, a través de los planes de estudio (en asignaturas cursadas o trabajo/proyecto fin de grado o máster) o mediante actividades extracurriculares.

Dentro de la primera línea de trabajo se incorporó, durante el cuatrimestre pasado, la competencia transversal CT05: Diseño y proyecto, dentro de la asignatura de Tecnología Hidráulica del Master en Ingeniería Agronómica.

El Instituto de Ciencias de la Educación junto con el Vicerrectorado de Estudios, Calidad y Acreditación de la UPV, describen esta competencia como aprender a "diseñar, dirigir, desarrollar y evaluar una idea de manera eficaz hasta concretarla en un servicio o producto".

La asignatura de Tecnología Hidráulica (5 ETCS) permite trabajar esta competencia a través de la metodología activa aprendizaje basado en proyectos (AbP), descrita en el programa ERAGIN de la Universidad del País Vasco (web Servicio Asesoramiento Educativo UPV/EHU), donde los alumnos en un grupo, diseñan y desarrollan un proyecto de ingeniería dentro del campo de la hidráulica agrícola.

De esta forma, el estudiante afronta un problema dentro de un escenario real que debe estructurar, y esforzarse, por encontrar soluciones para resolverlo (Johnson et al. 2000).

\section{Objetivos}

El objetivo principal de este artículo es presentar la experiencia docente llevada a cabo con los alumnos del Master de Ingeniería Agronómica, y analizar posibles mejoras en su implementación de cara al siguiente curso académico.

Además, la experiencia también permite evaluar el grado de adquisición de esta competencia, y colateralmente, las de (CT-08) Comunicación efectiva y (CT-06) Trabajo en grupo y liderazgo. 


\section{Desarrollo del proyecto}

\subsection{Metodología}

El aprendizaje basado en proyectos plantea un problema como punto inicial. En este caso, es un problema común que consiste en el desarrollo y redacción de un proyecto simplificado de una instalación de bombeo de agua dentro de las obras de infraestructura de una red colectiva de riego de una Comunidad de Regantes (CR).

El trabajo es en grupos de 3 alumnos al azar establecidos por los profesores de la asignatura. Consecuentemente, el alumno trabaja en un equipo con miembros no conocidos lo que permite potenciar su habilidad social y de liderazgo dentro del grupo.

Además, el problema es común para todos pero cada grupo tiene un escenario e información de partida diferente para su resolución. De esta forma, los profesores evitan la resolución conjunta del problema y favorecen el trabajo independiente de cada grupo.

Los profesores mediante tutorías, materiales docentes y herramientas de cálculo guían a los alumnos en el desarrollo del trabajo, pero es el mismo grupo de trabajo quien dirige su aprendizaje.

Para ello y como guía del proyecto, se establecen una serie de fases que permiten el seguimiento del mismo y su planificación. La finalización de cada una de estas fases es un punto de control para los profesores, que son capaces de valorar si los estudiantes logran desarrollar esa etapa satisfactoriamente, o por el contrario requieren de alguna puntualización o corrección que mejore sus resultados.

La duración establecida para el trabajo es de 6 semanas y el cronograma entregado a los alumnos se indica en el siguiente epígrafe.

\subsection{Fases del proyecto}

\subsubsection{Fase 1}

Buscar y ordenar la información de partida necesaria para el diseño de la impulsión y establecer las estrategias de funcionamiento del sistema, de tal forma, que se cubran las necesidades hídricas de los cultivos que se pretende regar (1 semana).

\subsubsection{Fase 2.}

Los alumnos deberán aplicar los procedimientos de diseño y dimensionado de las infraestructuras hidráulicas, a partir de los conocimientos adquiridos en la asignatura, en base a criterios funcionales y de optimización técnico económica con un aprovechamiento eficiente de recursos disponibles (hídricos y energéticos) (2 semanas).

\subsubsection{Fase 3.}

Analizar otras alternativas, y seleccionar y justificar una viable y adecuada. Cuantificar económicamente la solución adoptada.

(cc) EY-NC-ND 2016, Universitat Politècnica de València

Congreso In-Red (2016) 
Redactar el proyecto con todos sus documentos (3 semanas).

En la Fig. 1 se resumen estas fases.

\section{Fase 1 ( 1 semanas) documentación para cubrir los requisitos establecidos.}

Fase 2 ( 2 semanas)

\section{Fase 3 ( 3 semanas)}

Diseñar y dimensionar el sistema.

Analizar otras alternativas y seleccionar y justificar la elegida.

Buscar información de partida, Redactar el proyecto técnico.

Fig. 1 Fases del proyecto.

\subsection{Requisitos mínimos del proyecto final}

El desarrollo de esta competencia favorece que el alumno integre conocimientos de diversas disciplinas, promoviendo el trabajo autónomo del grupo y autodirigiendo su propio proyecto.

La planificación del mismo y el ajuste del tiempo en cada fase la realiza el propio equipo, asumiendo una serie de plazos indicados tras la resolución de cada fase con la entrega de documentos parciales.

No obstante, no se debe perder de vista que es una actividad docente, y en cierta medida guiada, con lo que se exige de unos requisitos mínimos en la resolución y redacción del proyecto. Estos requisitos se describen, a continuación, en la Tabla 1.

Tabla 1. Requisitos mínimos del proyecto

\section{Documento $n^{0} 1$ : Memoria y Anejos a la memoria.}

\begin{tabular}{ll}
\hline Anejos mínimos & Datos climáticos \\
\cline { 2 - 2 } & Determinación de los parámetros de riego. Diseño agronómico. \\
\hline Diseño y dimensionado de la impulsión. \\
\hline $\begin{array}{l}\text { Selección de los equipos de bombeo. Punto de funcionamiento } \\
\text { y regulación. }\end{array}$
\end{tabular}

Documento $n^{0}$ 2: Planos.

\begin{tabular}{ll}
\hline Planos & Ubicación \\
\cline { 2 - 2 } Planta general de la impulsión. \\
\cline { 2 - 2 } Perfiles longitudinales de la impulsión. \\
${ } }$
\end{tabular}

\section{Documento no 3: Presupuesto simplificado}

Valoración económica que incluya un mínimo de unidades de obra, mediciones, presupuestos parciales y presupuesto general.

(cc) EY-NC-ND 2016, Universitat Politècnica de València

Congreso IN-RED (2016) 


\subsection{Recursos utilizados y seguimiento tutorial.}

El desarrollo de la competencia requiere de un apoyo y seguimiento por parte de los profesores. Por ello, se ha facilitado a los alumnos la información y los recursos apropiados.

- Para el trazado en planta de la impulsión se tomará como base cartográfica las de plataformas SIGPAC, SIGNA o Google Maps.

- $\quad$ La selección de equipos de bombeo se debe realizar buscando información técnica en páginas web de los fabricantes de bombas vistos en la asignatura.

- La tarifa eléctrica a contratar será la 3.1.A., con precios de los términos de energía y potencia que pueden consultarse en webs de las principales compañías suministradoras de energía eléctrica (Iberdrola, Endesa, Edp, etc).

El tratamiento de la información y los correspondientes cálculos pueden realizarse con las aplicaciones informáticas vistas durante la asignatura (DISEÑO AGRONÓMICO RL, ASHIDRAR) o en otras disciplinas de grado o master.

\subsection{Evaluación del proyecto}

Como procedimiento de evaluación de la competencia transversal CT-05 Diseño y proyecto, se solicita la redacción de un informe escrito en cada una de las fases, que finalizará con el documento final, con los pesos y plazos fijados en la Tabla 2.

Tabla 2. Entregables, pesos y plazos de cada etapa.

\begin{tabular}{lcc}
\hline Entregables escritos & $\begin{array}{l}\text { Peso calificación } \\
\text { documento escrito }\end{array}$ & Fecha entrega \\
\hline $\begin{array}{l}\text { Documento 1. Tratamiento de la } \\
\text { información y diseño agronómico } \\
\text { y condiciones de funcionamiento }\end{array}$ & $25 \%$ & 4 diciembre 2015 \\
\hline $\begin{array}{l}\text { Documento 2: Diseño y } \\
\text { dimensionado de a impulsión. }\end{array}$ & $25 \%$ & 23 diciembre 2015 \\
\hline $\begin{array}{l}\text { Documento 3: Diseño final: } \\
\text { Proyecto simplificado }\end{array}$ & $40 \%$ & \multirow{2}{*}{15 enero 2015 } \\
\hline Verificación solución alternativa. & $10 \%$ & \\
\hline
\end{tabular}

Como se observa, se ponderan con mayor peso los resultados de aprendizaje que demuestran categorias de orden mayor en la Taxonomía revisada de Bloom (Anderson, 2001), como puede ser analizar el diseño final y evaluar posibles alternativas.

Asimismo, cada equipo realiza una defensa que consiste en una breve exposición oral de unos 10 a 15 minutos de la solución adoptada que se evaluará mediante la rúbrica de la Fig.2. Posteriormente los tres integrantes del grupo, indistintamente, deberán contestar a cuantas cuestiones se les formulen sobre el trabajo por el profesorado de la asignatura. El peso del documento final será del $60 \%$ y de la defensa del mismo del $40 \%$.

(cc) EY-NC-ND 2016, Universitat Politècnica de València

Congreso In-Red (2016) 


\begin{tabular}{|c|c|c|c|c|c|}
\hline \multicolumn{6}{|c|}{ Rúbrica para la defensa del proyecto : EXPOSICIÓN ORAL } \\
\hline \multirow[b]{2}{*}{ Partes } & \multirow[b]{2}{*}{ Indicador } & \multicolumn{4}{|c|}{ Descriptor } \\
\hline & & No & $\begin{array}{c}\text { En } \\
\text { desarrollo }\end{array}$ & $\begin{array}{c}\text { Bien/ } \\
\text { adecuado }\end{array}$ & Excelente \\
\hline \multirow{3}{*}{ CONTENIDO DEL PROYECTO } & $\begin{array}{l}\text { La exposición del proyecto está estructurada de modo } \\
\text { coherente, explicando de forma ordenada la solución } \\
\text { adoptada. }\end{array}$ & & & & \\
\hline & $\begin{array}{l}\text { El contenido es adecuado y el alumno justifica } \\
\text { correctamente el diseño propuesto. }\end{array}$ & & & & \\
\hline & $\begin{array}{l}\text { El alumno se ajusta al tiempo y transmite la información } \\
\text { más relevante. }\end{array}$ & & & & \\
\hline \multirow{3}{*}{$\begin{array}{l}\text { EXPOSICIÓN, ACTITUD Y } \\
\text { MEDIOS DE APOYO }\end{array}$} & $\begin{array}{l}\text { Muestra una disposición personal favorable en la } \\
\text { exposición. }\end{array}$ & & & & \\
\hline & $\begin{array}{l}\text { Utiliza el lenguaje con corrección y transmite seguridad y } \\
\text { tranquilidad. }\end{array}$ & & & & \\
\hline & $\begin{array}{l}\text { Los medios de apoyo utilizados, tanto en el desarrollo del } \\
\text { proyecto como en la defensa, son adecuados y con un } \\
\text { formato cuidado. }\end{array}$ & & & & \\
\hline
\end{tabular}

Fig. 2 Rubrica defensa oral del proyecto.

\section{Resultados}

Los resultados de esta primera experiencia en la asignatura de Tecnología Hidráulica han sido bastante satisfactorios. En algunos equipos se ha observado, claramente, descoordinación y falta de planificación, pero en general, el aprendizaje basado en proyectos, permite la adquisición y evaluación de la competencia CT-05 definida por la UPV.

La valoración de los alumnos tras la actividad en la competencia CT-05 utilizando la escala A (excelente), B (adecuado), C (en desarrollo) y D (no alcanzado) propuesta por la UPV, muestra que el $27 \%$ obtuvo el nivel de excelente, un 59\% adecuado, un $12 \%$ en desarrollo y un $2 \%$ no alcanzó el nivel adecuado por no presentar el proyecto.

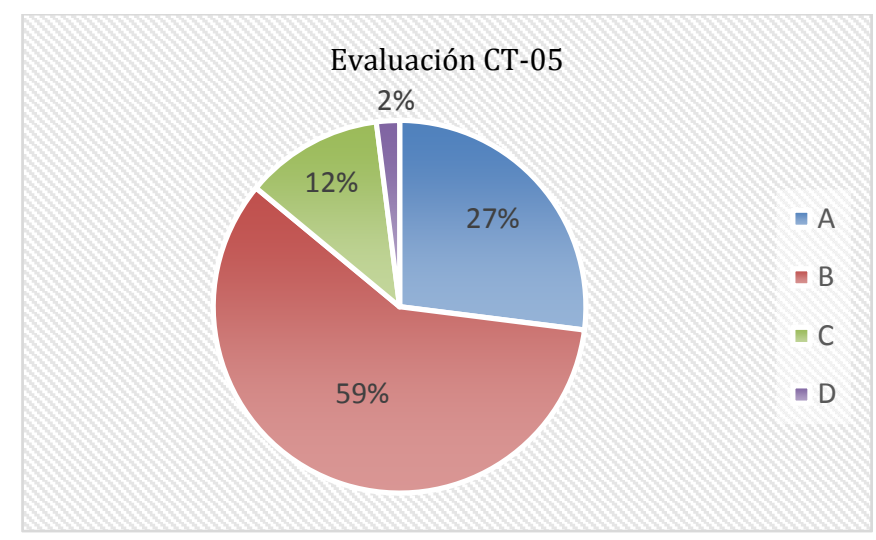

Fig. 3 Gráfico circular de evaluación de la CT-05 Diseño y proyecto.

Además, se realizaron entrevistas a los alumnos para conocer su opinión acerca de la propuesta de proyecto, $\mathrm{y}$ las respuestas fueron positivas con algunas aportaciones interesantes que se implementarían el curso próximo.

(cc) EY-NC-ND 2016, Universitat Politècnica de València 
Los alumnos manifestaron su descontento durante el trabajo en equipo, con desequilibrios claros en la carga y distribución de tareas, que posteriormente no se tenían en cuenta en la evaluación. La propuesta de mejora en este sentido, es introducir una evaluación entre iguales, es decir, entre los miembros del equipo para detectar de esta forma estos posibles desequilibrios y evitar, a su vez, actitudes parásitas en el equipo.

También, reprocharon que el inicio del proyecto se retrasó en exceso y se cogieron festivos que impidieron un seguimiento tutorial más adecuado.

Por el contrario, mostraron una opinión positiva hacia la actividad desarrollada indicando que el trabajo autónomo y el compendio de disciplinas que abarcaba les había dado una visión global del problema planteado muy real y cercano a su futura vida profesional.

\section{Conclusiones}

En este artículo se muestra la experiencia llevada a cabo en la asignatura de master de Ingeniería Agronómica para la adquisición y evaluación de la competencia transversal CT-05. Los resultados han sido satisfactorios y el grado de implicación y rendimiento de los alumnos alto.

Se concluye con una serie de propuestas de mejora para implementar en el próximo curso tras el análisis de resultados y las entrevistas a los alumnos al finalizar la actividad.

Las mejoras propuestas introducen una evaluación del proyecto adicional entre los alumnos de un mismo grupo, con el objetivo de corroborar el trabajo y la planificación de tareas dentro del equipo. También, se incorporarán tutorías grupales que estimulen el debate y diálogo sobre dudas que surjan durante el desarrollo del proyecto.

Desde el punto de vista de los profesores, la experiencia docente ha sido gratificante ya que se ha trabajado con algunos grupos muy motivados y con gran interés por la materia. Además, la introducción de un proyecto como trabajo académico final ha permitido tener una visión global de la asignatura, requiriendo en algunas fases conocimientos previos de otras materias, con la consecuente búsqueda de información.

\section{Referencias}

ANDERSON, L. W. AND KRATHWOHL, D. R., et al. (Eds..) (2001) A Taxonomy for Learning, Teaching, and Assessing: A Revision of Bloom's Taxonomy of Educational Objectives. Allyn \& Bacon. Boston, MA (Pearson Education Group).

FERNADEZ MARCH, A (2010). La evaluación orientada al aprendizaje en un modelo de formación por competencias en la educación universitaria. REDU: Revista de Docencia Universitaria, ISSN 1887-4592, Vol. 8, №. 1, 2010, 35 págs.

JOHNSON, D.W., JOHNSON,R.T.,and SMITH, K.A., (2000). Active Learning: Cooperation in the College Classroom. Interaction Book, Edina, MN.

TARDIF, J. (2006). L'évaluation des compétences. Documenter le parcours de développement. Montréal : Chenelière Éducation.

(cc) EY-NC-ND 2016, Universitat Politècnica de València

Congreso In-Red (2016) 
UNIVERSITAT POLITÈCNICA DE VALENCIA. Proyecto Institucional sobre Competencias Trasnversales.

\section{Libro electrónico}

GUISASOLA, J; GARMENDIA, M. (2014) El programa ERAGIN de formación en metodologíasactivas de la UPV/EHU. Aprendizaje basado en problemas, proyectos y casos: diseño e implementación de experiencias en la universidad. Eds. Universitad del Pais Vasco.

GONZALEZ, J., y WAGENAAR, R. (2003). Tuning Educational Structures in Europe. Informe final - fase uno. Bilbao: Universidad de Deusto. http://www.unideusto.org/tuningeu/images/stories/documents/General_Brochure_Spanish_version.pdf

\section{Software}

DISEÑO AGRONÓMICO RL (2015). J Arviza.

ASHIDRAR, (2015) J Arviza.

Páginas web

PORTAL UNIVERSIDAT POLITECNICA DE VALENCIA < http://competenciast.webs.upv.es/>.

SERVICIO ASESORAMIENTO EDUCATIVO, UNIVERSIDAD DEL PAIS VASCO, (UPV/EHU) Programa Eragin $<$ http://www.ehu.eus/es/web/sae-helaz/eragin-irakaskuntzametodologia-aktiboak>

ESCUELA TÉCNICA SUPERIOR DE INGENIERÍA AGRONÓMICA Y DEL MEDIO NATURAL (2013). Master en Ingeniería Agronómica. < https://www.upv.es/entidades/ETSIAMN/index-va.html> 